\title{
ReviewArticle
}

\section{EBOLA VIRUS THREAT: AWARENESS, EDUCATION AND PREPAREDNESS}

\section{Muhammad Zubair Tahir}

\author{
Correspondence: Muhammad Zubair Tahir, Public Health Consultant, \\ FANA Global Consultancy and Business Limited, 1 St. Agatha's Road, Ward End, \\ Birmingham, B8 2TU, United Kingdom. E-mail: zubair01pk@yahoo.com. Phone: +44 \\ 7448343299
}

\footnotetext{
Abstract

Background: Ebola virus (EBOV) is a real threat and Ebola virus disease (EVD) can be transferred to any country. International flights in a country enhance the risk of an EBOV outbreak and its spread, especially in thickly populated cities. There is a great need for awareness among common persons, health professionals and decision-makers about EVD and preparedness to manage an outbreak. Objective: The main objective of the study was to describe Ebola virus characteristics, mode of spread, risk factors, signs and symptoms, persistence, and preventions.

Methods: PubMed was searched with keywords, and 700 articles were found. Abstracts of all searched articles were reviewed. WHO and CDC websites were explored for relevant information.

Results: Contact with EVD patient's body fluids is the strongest risk factor. Although the incubation period of EBOV is from 2 to 21 days, while usually symptoms are developed between 4 and 10 days after exposure of Ebola virus. Similarities of signs and symptoms with other infectious diseases, mostly lead misdiagnosis. EBOV proteins reduce the human immune system's response to viral infections. In EVD survivors, Ebola virus can remain in semen, breast milk, ocular (eye) fluid, and spinal column fluid. It is very difficult to control and manage an outbreak of Ebola virus in areas of high population density areas.

Conclusion: Awareness, knowledge, and education about EVD among populations and clinicians can decrease fear, risk, and fatalities. Preparedness and training to HCWs can minimise the risk of EVD especially in thickly populated cities and areas of a country.

Keywords: Ebola virus, spread, fear, risk factors, history
} 


\section{Introduction}

Zoonotic outbreaks and the appearance of new pathogens have appeared throughout time and it will continue as nature develops new microorganisms (1). In 1976, two similar viral diseases outbreaks, simultaneously, were reported in Africa at different places. One was in Nzara, South Sudan and the other in a Yambuku village, near the Ebola River, in the Democratic Republic of Congo. Due to the Ebola river, the viral disease took the name Ebola virus disease (EVD) (2).

EVD most commonly affects humans and primates, monkeys, gorillas, and chimpanzees. There are three genera of the virus family Filoviridae: Cuevavirus, Marburgvirus, and Ebolavirus. The genus Ebolavirus identified species include Zaire, Bundibugyo, Sudan, Reston, Bombali and Taï Forest. Of these, Ebola, Sudan, Taï Forest, and Bundibugyo viruses are known to cause EVD in people (3).

Ebola virus reservoir has not been indubitably proven (4). It is assumed that the reservoir for Ebola virus (EBOV) is in bats as serological evidence has been found in several bat species (5). There is evidence of EBOV presence in three species of fruit bats Hypsignathus monstrosus, Epomops franquetiand and Myonycteris torquata, which indicates that these animals may be primary sources and carriers of EBOV (6)(7).

From African countries like Guinea, Liberia, Sierra Leone, and Nigeria, EBOV has been transferred to the other countries in Europe and American regions through the travellers (7). The imported cases of EVD among healthcare workers (HCWs) in Europe and the United States indicates that they need more education and training to protect themselves and others from Ebola virus infection. Clinicians must have knowledge about the clinical picture of EVD, differential diagnosis and therapeutic approach (9)

Ebola infection outbreak 2014-16 was difficult to control as it was in areas of high population density. Previous outbreaks were easier to control as they were in rural areas, where isolation is easier. If the virus spreads to developing countries with larger cities, where millions of people live in very densely populated areas, like Bangkok, Cairo, Dhaka or Mumbai then it will be very difficult to control its spread and fatalities. Therefore, the preventive measures should be an education delivery about EVD among populations, clinicians and HCWs (10).

International flights in any country mean that there is a great risk of an EBOV outbreak and spread (11). The largest EBOV outbreak, from December 2013 to January 2016, was in West Africa which resulted in 28,616 EVD cases and 11,310 deaths (12). On 1st August 2018, in the Democratic Republic of Congo, the ministry of health reported an EVD outbreak in North Kivu province. Since then over 4,100 people have been in contact with EBOV infected persons, 81 people died with EVD and more than 2,300 people are being followed up by the ministry of health (13).

Ebola virus can spread not only in the developed world but also in developing countries (14). It can erupt in any country and spread fiercely in thickly populated cities due to travellers, lack of information among people and unpreparedness in all sectors. There is a great need to educate people and increase knowledge about EVD through awareness campaigns, print, electronic, and social media (15). The aim of the study is to describe Ebola virus characteristics, mode of spread, persistence, symptoms, signs, and preventions.

Methodology

The main search was made at PubMed with keywords "Ebola viru AND history", "Ebola virus AND risk factors", "Ebola virus AND spread", "Ebola virus AND fear", and 700 articles were found. The search had no time limit, filtered by English language and humans only. All searched articles were reviewed for all information about Ebola virus characteristics, transmission, symptoms and signs, risks, persistence, and preventions.

Inclusion criteria for PubMed search included: original studies, review studies, case reports, systematic reviews, meta-analysis and clinical studies. The exclusion criteria included published articles and reports which do not qualify inclusion criteria, all unrelated researches, and unclear research results. Abstracts of all 700 articles were reviewed, and only 42 research articles were selected for the study.

An additional search was made with keyword "Ebola virus" at World Health Organisation and The Centers for Disease Control and Prevention (CDC) websites for the latest facts and guidelines. The search was started on 3rd October 2018 and all search along with review process were completed on 9th October 2018.

\section{Results}

Structure and characteristics of Ebola virus

The diameter of viron is about $80 \mathrm{~nm}$ and its length ranges from 970 to $1200 \mathrm{~nm}$. The viral genome length is approximately $19 \mathrm{~kb}$ and it is the longest among all viruses belonging to Mononegavirales (16). In Ebola virus infection, initially, the virus infects macrophages and endothelial cells of the body. It mainly produces a Viral Protein 24 (eVP24) which prevents interferon-based signals which are essential for the destruction of Ebola viruses (8)

In recent years, knowledge about EBOV fundamental principles of replication and pathogenesis has been significantly improved due to research. According to the latest research, it is found that each viral protein plays a specific role and proteins have large numbers of overlapping roles during virus replication (17).

The interferon (IFN) system can effectively control Ebola virus spread in infected mice but is not effective in infected humans due to viral proteins VP24 and VP35. These viral proteins play the main role in suppressing the IFN response. EBOV proteins reduce the human immune system's response to viral infections by interfering with the cells' ability to produce and respond to interferon proteins such as interferon-alpha, interferon-beta, and interferon gamma (18).

\section{The spread of the Ebola virus}

Ebola virus was transmitted from wild animals to humans and then spread amongst people through human-to-human transmission. The virus enters a human through close contact with the blood, secretions, organs or other bodily fluids of EBOV infected animals like chimpanzees, gorillas, fruit bats, monkeys, forest antelope and porcupines ill or dead (2).

Ebola virus later spreads in the human population through human-tohuman transmissions; (a) by direct contact, broken skin or mucous membranes with the virus infected people blood, secretions or other bodily fluids, (b) during burial of infected dead body, due to direct contact, (c) through direct contact with contaminated surfaces and materials like bedding, clothing etc (2).

EVD survivors carry EBOV in semen for over 12 months, cause sexual transmission and in breast milk which can transmit infection in infants due to breastfeeding (19). The spread of EVD is accelerated by poor information on prevention and treatment, insufficient medica facilities, lack of clinician's knowledge, poor sanitation, travel and unsafe burial practices at outbreak sites (20).

EBOV, usually, is not transmitted by food but it may spread through the handling and consumption of bushmeat (wild animals hunted for food) There is no recorded evidence that mosquitoes or other insects can transmit Ebola virus (3).

\section{Fear and risks of Ebola virus spread}

Contact with EVD patient's body fluids is the strongest risk factor for EBOV transmission and onset of the disease (21). Knowledge and information about causes and preventions of EBOV amongst populations can minimise the risk and fear of EVD. There is a great need to deliver information and knowledge to clinicians and HCWs about the clinical presentation of EVD, diagnosis, treatment, mode of spread and prevention. It will decrease an irrational fear among clinicians and HCWs (22).

Hospitals staff have greater exposure to EBOV, so the fear of the EVD spread is common among them (23). Nurses, midwives, and other medical staff faced increased risks of getting Ebola virus infection, during the Ebola virus epidemic in health facilities, but they continued to provide essential health care. Proper knowledge about EVD can decrease their fear and training will have a significant impact on the control of EVD spread (24).

In order to reduce the risk of imported Ebola virus disease and its spread in a country, there is a need of proper surveillance and monitoring system to track suspected cases and save the general public from fear and EVD by timely appropriate interventions (25).

Symptoms, signs, and diagnosis of EVD

According to the World Health Organisation (WHO) EVD guidelines, the incubation period of EBOV is from 2 to 21 days, but initial symptoms usually appear between 4 and 10 days (2). Currently, there is no evidence that sex of female or male increases Ebola virus transmission and vulnerability. The transmission and onset of the disease are only related to the level of exposure to Ebola virus (26).

Symptoms and signs

WHO has issued guidelines for clinicians about EVD signs, symptoms, diagnosis, lab test etc. Initial symptoms are sudden fever, 
fatigue, muscle pain, headache, and sore throat. At a later stage vomiting, diarrhoea, rash, and in some cases, both internal and external bleeding (e.g. oozing from the gums, blood in the stools) can be presented. The early clinical presentation of Ebola virus infection has similarities with other infectious diseases, which causes misdiagnosis and creates differential diagnosis difficulties. EVD has symptomatic treatment (2).

\section{Diagnosis for EVD}

Laboratory findings include low white blood cells, a decrease in platelets counts and elevated liver enzymes. Confirmatory tests for Ebola virus infection include:

o Antibody-capture enzyme-linked immunosorbent assay (ELISA)

o Antigen-capture detection test

o Serum neutralization test

o Reverse transcriptase polymerase chain reaction (RT-PCR) assay

o Electron microscopy

V Virus isolation by cell culture (2)

\section{Currently WHO recommended test}

o Automated or semi-automated nucleic acid test (NAT)

o Rapid antigen detection test, for screening purposes, for use in remote settings where NATs are not readily available (2).

The preferred specimens for diagnosis of $E V D$

o The whole blood sample collected in ethylenediaminetetraacetic acid (EDTA)

o Oral fluid specimen when blood collection is not possible

Samples collected have an extreme biohazard risk, so laboratory testing should be conducted under maximum biological containment conditions. All collected specimens should be packaged using the triple packaging system for transportation (2).

\section{Persistence of Ebola virus}

Ebola virus persists in some EVD survivors in immune-privileged sites like testicles, inside the eye and the central nervous system. In infected women during pregnancy, the virus persists in the placenta, amniotic fluid, and foetus. While in women who get Ebola virus infection during breastfeeding, the virus may persist in breast milk (2).

In EVD survivors, Ebola virus can remain in semen, breast milk, ocular (eye) fluid, and spinal column fluid. On doorknobs and countertops, the virus can survive for several hours and in blood, the virus can survive up to several days at room temperature (3).

Sexual activity by infected male survivors was the most likely route of transmission confirmed or suspected in half of the outbreaks. The case reports from the Ebola virus fourth outbreak (Sierra Leone) and the fifth outbreak (Guinea) advocate that EVD survivors' infectious semen might have infected healthy humans without sexual contact like the transmission of EBOV through small lesions (27).

A case report from the West African outbreak in 2015 was the first convincing evidence of male to female sexual transmission of the Ebola virus. Available information suggests that sexual transmission of Ebola virus is a relatively rare event. However, persistent infection of Ebola virus in body with unprotected sexual activity can possibly lead to EVD outbreak (28).

\section{Prevention and contro}

Ebola virus infection spread, in population, can be prevented with early case detection, community engagement, contact tracing, immediate patient isolation, clinical care, and proper infection control measures including safe burial of EVD persons. There are risks of human Ebola virus infections from animals and from EVD survivors, but they can be minimised/controlled by proper information and knowledge delivery to communities about the disease spread (29).

\section{WHO recommendations}

\section{Male EVD survivors should;}

o Abstain from all types of sex, or use a condom

o Have semen testing after 3 months, and if the male survivor has semen test positive for Ebola virus then he should have the test every month till semen test becomes negative for virus twice by

RT-PCR. There should be an interval of one week between tests by RT-PCR

o Receive counselling to ensure safe sexual practices (2).

After the discovery of EVD cases, a rapid response by health and other authorities can prevent transmission of the disease and minimise the risk of a major epidemic in large populations. Therefore, there is a need to ensure that populations have sufficient knowledge about EVD and health services are ready for EVD outbreak management (30). In EVD outbreaks, for timely control, there are requirements of community leaders' involvement, isolation of infected persons, trained, qualified and properly equipped health staff to manage the patients and rapid burial of infected dead person (31).

During management of EBOV outbreak, occupational exposure to blood and other body fluids are due to lack of use of personal protective equipment and needlestick or sharp injuries which result in occupational Ebola virus disease (OEVD). Prevention of OEVD should be one of the top priorities during EVD outbreak and it is possible by the transfer of knowledge about EVD, training for protection, preparedness, and management of Ebola virus outbreak (32).

\section{Vaccination}

After clinical trials conducted in Africa, Europe, and the US, the most advanced first-generation Ebola vaccines rVSV-ZEBOV and ChAd3ZEBOV have been found safe. A single dose of rVSV-ZEBOV or ChAd3-ZEBOV has the ability to generate protective antibodies against Ebola virus (33). In a trial conducted in Guinea in 2015 by WHO and other international partners, rVSV-ZEBOV vaccine was found to be highly protective against the virus (2) (34).

Discussion

In this review, knowledge and the latest information about EBOV characteristics, different modes of spread, signs and symptoms, management, control/prevention, sustainability and vaccination of EVD would be beneficial. The risk of EVOV outbreak and its spread is increased when populations, clinicians, HCWs, health, and other authorities have lack of knowledge and unpreparedness.

In humans, EVD is associated with infected wildlife copses or remains contact, so identifying great ape outbreaks would have an impact to control EBOV outbreak and fatality rates (35). Creating awareness and providing knowledge through educational materials, health education among communities about EVD and training programmes among health care workers (HCWs) would be helpful to manage outbreaks for epidemic management (36) (37). Community involvement in EVD outbreak would be a better strategy to control EBOV epidemic (38). A study aimed to find clinical signs, symptoms and risk factors for fatal outcome of 2013-2016 outbreak of EVD in West Africa revealed important predictors of EVD deaths; shorter time from onset of symptoms to admission, high viral load, severe pain, bloody faeces and diarrhoea and development of other bleeding manifestations during hospital stay (39). Fears and misperceptions due to lack of information about EVD and lack of trust in the health care system could be the possible factors of people's delayed care seeking behaviour during EBOV outbreak in Sierra Leone in 2014-15 (40).

Ebola virus-infected patients' environment is an important risk factor of its spread. HCWs are highly exposed to EBOV due to contact with EVD patients. In a study, samples were taken with swabs from very close areas of EVD patients like surfaces of an ETU and personal protective equipment of $\mathrm{HCWs}$ after patient care. It was found that there was the persistence of EBOV RNA around the environment of EVD patients and of HCWs, which shows that EVD patients' environment is a potential source of Ebola virus contamination (41).

EBOV presence in semen of EVD survivors is also a hazard. The virus can be detected in semen up to 18 months after getting EVD and it shows the biggest risk of EBOV reappearance in the communities. Auspiciously, sexual transmission of Ebola virus is rare. It is very interesting that EBOV female-to-male sexual transmission has not been identified (27)

The latest study of EVD survivors shows the long-term presence of Ebola virus RNA in semen and it declined with the passage of time. It was found that after 13 to 15 months $11 \%$ and after 16 to 18 months $4 \%$ person semen specimens showed EBOV RNA. While at 19 months or later no EBOV RNA was detected in semen of EVD survivors (42).

Breastfeeding is a potential risk of EVD spread. In August 2015 in Guinea, a 9-month-old infant died from EVD and laboratory investigations showed persisting EBOV RNA in father's seminal fluid and the mother's breast milk. The genomic analysis strongly suggests that Ebola virus transmission was from mother to the child through breastfeeding (43).

A study contradicts sexual transmission of EBOV. EVD cases in the previous 20 EVD outbreaks in West Africa were not by sexual transmission. Although Ebola virus was found at sites and in fluids related to sexual activities, this appears at different stages of the 
disease. Ebola virus in the recovering period appears in rectum, vagina and in semen being the longest of up to at least 101 days. It concluded that there is no evidence of sexual transmission of Ebola virus and it is an only theoretical probability. Hence, it was recommended that those recovering from EVD must abstain from all physical sexual activities or use condoms for 3 months after the onset of symptoms (44). Presence of the virus is a risk factor.

Ebola virus has been detected in dogs and cats in areas of Ebola virus outbreaks, but there is no evidence or documented report that dogs or cats can spread EBOV to people or other animals. Only pigs have the risk of getting EVD (3).

The new risk of fatal EBOV spread is through terrorists. It might be difficult that terrorists get a filovirus to create fear and panic (45). Ebola virus infection initially disables the immune system and later disables the vascular system which leads to haemorrhage, hypotension, followed by shock and death. It is a perception that Ebola virus can be used, anywhere, as a bioterrorism agent for fear and panic (46).

There should be preparedness and response practices, according to guidelines, at local and regional levels for Ebola virus outbreaks management. In the Netherlands, with curative and public health sectors participation a national platform for preparedness has been established which may be helpful for other countries to follow (47).

EVD educational programmes and their evaluation by health and other authorities, in developing or developed countries, would minimise the risks of EBOV outbreaks and spread.

Conclusion

Awareness, knowledge, and education about Ebola virus disease among populations and clinicians can decrease fear, risk, and fatalities. Preparedness and training to health care workers can minimise the risk of EVD especially in thickly populated cities and areas of a country. Conflict of interest

None

References

Harrod KS. Ebola: history, treatment, and lessons from a new emerging pathogen. Am J Physiol Lung Cell Mol Physiol 2015; 308(4): L307-L313.

2. World Health Organisation. Ebola virus disease. h t t p : / / w w w. who.int/en/news - room/fact sheets/detail/ebola-virus-disease (accessed 01 October 2018).

3. Centers for Disease Control and Prevention. Ebola (Ebola virus disease). What is Ebola Virus Disease? https://www.cdc.gov/vhf/ebola/about.html (accessed 01 October 2018).

4. Zhang L, Wang H. Forty years of the war against Ebola. J Zhejiang Univ Sci B. 2014;15(9):761-5.

5. Pourrut X, Souris M, Towner JS, Rollin PE, Nichol ST, Gonzalez JP, et al. Large serological survey showing cocirculation of Ebola and Marburg viruses in Gabonese bat populations, and a high seroprevalence of both viruses in Rousettus aegyptiacus. BMC Infect Dis. 2009;9:159.

6. Leroy EM, Kumulungui B, Pourrut X, Rouquet P, Hassanin A, Yaba $\mathrm{P}$, et al. Fruit bats as reservoirs of Ebola virus. Nature. 2005 ;438(7068):575-6.

7. Laupland KB, Valiquette L. Ebola virus disease. Can J Infect Dis Med Microbiol. 2014;25(3):128-9.

8. Jadav SS, Kumar A, Ahsan MJ, Jayaprakash V. Ebola virus: current and future perspectives. Infect Disord Drug Targets. 2015;15(1):20-31.

9. Bociaga-Jasik M, Piatek A, Garlicki A. Ebola virus disease - pathogenesis, clinical presentation and management. Folia Med Cracov. 2014;54(3):49-55.

10. Kalra S, Kelkar D, Galwankar SC, Papadimos TJ, Stawicki SP, Arquilla B, et al. The emergence of Ebola as a global health security threat: from 'lessons learned' to coordinated multilateral containment efforts. J Glob Infect Dis. 2014;6(4):164-77.

11. Hisam A, Rana MN, Rahman M. Knowledge and attitude regarding Ebola virus disease among medical students of Rawalpindi: A preventable threat not yet confronted. Pak J Med Sci. 2016;32(4):1015-9.

12. World Health Organisation. Ebola data and statistics. http://apps.who.int/gho/data/view.ebola-sitrep.ebolasummary-20160316?lang=en (Accessed 03 October 2018).
13. Méd e c ins $\mathrm{S}$ a n s F r o n t i ères. E b o la https://www.msf.org.uk/issues/ebola (accessed 13 October 2018).

14. Stein RA. What is Ebola?. Int J Clin Pract. 2015;69(1):4958.

15. Salman M, Shehzadi N, Hussain K, Saleem F, Khan MT, Asif N, et al. Knowledge of Ebola virus disease among a university population: A cross-sectional study. Am J Infect Control. 2017;45(2):e23-e25

16. Zawili?ska B, Kosz-Vnenchak M. General introduction into the Ebola virus biology and disease. Folia Med Cracoy. 2014;54(3):57-65

17. Cantoni D, Rossman JS. Ebolaviruses: New roles for old proteins. PLoS Negl Trop Dis. 2018;12(5):e0006349.

18. Kühl A, Pöhlmann S. How Ebola virus counters the interferon system. Zoonoses Public Health. 2012;59 Suppl 2:116-31.

19. Nordenstedt H, Bah EI, De la MA, Barry M, N'Faly M, Barry M, et al.

Ebola Virus in Breast Milk in an Ebola Virus-Positive Mother with Twin Babies, Guinea, 2015. Emerg Infect Dis. 2016;22(4):759-60

20. Burd EM. Ebola virus: a clear and present danger. J Clin Microbiol. 2015;53 (1):4-8

21. Francesconi P, Yoti Z, Declich S, Onek PA, Fabiani M, Olango J, et al.Ebola hemorrhagic fever transmission and risk factors of contacts, Uganda. Emerg Infect Dis. 2003;9(11):1430-7.

22. Del Rio C, Guarner J. Ebola: Implications and Perspectives. Trans Am Clin Climatol Assoc. 2015;126:93 112.

23. Kinsman J. A time of fear: local, national, and international responses to a large Ebola outbreak in Uganda. Global Health. 2012 Jun;8:15.

24. Jones S, Sam B, Bull F, Pieh SB, Lambert J, Mgawadere F, et al. Even when you are afraid you stay: A qualitative study. Midwifery. 2017;52:19-26.

25. Chen T, Ka-Kit Leung R, Liu R, Chen F, Zhang X, Zhao J, et al. Risk of imported Ebola virus disease in China. Travel Med Infect Dis. 2014;12(6 Pt A):650-8.

26. Nkangu MN, Olatunde OA, Yaya S. The perspective of gender on the Ebola virus using a risk management and population health framework: a scoping review. Infect Dis Poverty. 2017;6(1):135

27. Subissi L, Keita M, Mesfin S, Rezza G, Diallo B, Van Guch S. Ebola Virus Transmission Caused by Persistently Infected Survivors of the 2014-2016 Outbreak in West Africa. J Infect Dis. 2018 Jun 18. doi 10.1093/infdis/jiy280. [Epub ahead of print]

28. Mate SE, Kugelman JR, Nyenswah TG, Ladner JT, Wiley MR, Cordier-Lassalle T, et al. Molecular Evidence of Sexual Transmission of Ebola Virus. N Engl J Med. 2015;373(25):2448-54

29. Pigott DM, Golding N, Mylne A, Huang Z, Henry AJ, Weiss DJ, et al. Mapping the zoonotic niche of Ebola virus disease in Africa. Elife. 2014;3:e04395-e04395.

30. Breman JG, Johnson KM. Ebola then and now. N Engl J Med. 2014;371 (18):1663-6.

31. Agua-Agum J, Allegranzi B, Ariyarajah A, Aylward R, Blake IM, Barboza P, et al. After Ebola in West Africa-Unpredictable Risks, Preventable Epidemics. N Engl J Med. 2016Aug 11;375(6):587-96.

32. Ngatu NR, Kayembe NJ, Phillips EK, Okech-Ojony J, Patou-Musumari M, Gaspard-Kibukusa M, et al. Epidemiology of ebolavirus disease (EVD) and occupational EVD in health care workers in Sub-Saharan Africa: Need for strengthened public health preparedness. J Epidemiol. 2017;27(10):455-461.

33. Pavot V. Ebola virus vaccines: Where do we stand? Clin Immunol. 2016; 173:44-49.

34. CDC. Ebola (Ebola virus disease).Prevention. https://www.cdc.gov/vhf/ebola/prevention/index.html (accessed 12 October 2018). 
35 .

Reed PE, Mulangu S, Cameron KN, Ondzie AU, Joly D, Bermejo $\mathrm{M}$, et al. A new approach for monitoring ebolavirus in wild great apes. PLoS Negl Trop Dis. 2014;8(9):e3143.

36. Nyakarahuka L, Skjerve E, Nabadda D, Sitali DC, Mumba $\mathrm{C}$, Mwiine FN, et al. Knowledge and attitude towards Ebola and Marburg virus diseases in Uganda using quantitative and participatory epidemiology techniques. PLoS Negl Trop Dis. 2017;11(9):e0005907.

37. Khalafallah MT, Aboshady OA, Moawed SA, Ramadan MS. Ebola virus disease: Essential clinical knowledge. Avicenna J Med. 2017;7(3):96-102.

38. Pellecchia U, Crestani R, Decroo T, Van den Bergh R, AlKourdi Y. Social Consequences of Ebola Containment Measures in Liberia.PLoS One. 2015;10 (12):e0143036.

39. Haaskjold YL, Bolkan HA, Krogh KØ, Jongopi J, Lundeby $\mathrm{KM}$, Mellesmo S, et al. Clinical Features of and Risk Factors for Fatal Ebola Virus Disease, Moyamba District, Sierra Leone, December 2014-February 2015. Emerg Infect Dis. 2016;22(9):1537-44

40. Yamanis T, Nolan E, Shepler S. Fears and Misperceptions of the Ebola Response System during the 2014-2015 Outbreak in Sierra Leone. PLoS Negl Trop Dis. 2016;10(10):e0005077.
41.

Palich R, Irenge LM, Barte E, Augier A, Malvy D, Gala JL. Ebola virus RNA detection on fomites in close proximity to confirmed Ebola patients; N'Zerekore, Guinea, 2015. PLoS One. 2017;12(5):e0177350.

42. Deen GF, Broutet N, Xu W, Knust B, Sesay FR, McDonald SLR, et al. Ebola RNA Persistence in Semen of Ebola Virus Disease Survivors - Final Report. N Engl J Med. 2017 ;377(15):1428-1437.

43. Sissoko D, Keïta M, Diallo B, Aliabadi N, Fitter DL, Dahl BA, et al. Ebola Virus Persistence in Breast Milk After No Reported Illness: A Likely Source of Virus Transmission From Mother to Child. Clin Infect Dis. 2017;64(4):513516.

44. Rogstad KE, Tunbridge A. Ebola virus as a sexually transmitted infection. Curr Opin Infect Dis. 2015;28(1):835.

45. Bray M. Defense against filoviruses used as biological weapons. Antiviral Res. 2003;57(1-2):53-60.

46. Ansari AA. Clinical features and pathobiology of Ebolavirus infection. J Autoimmun. 2014;55:1-9.

47. Swaan CM, Öry AV, Schol LGC, Jacobi A, Richardus JH, Timen A. Ebola Preparedness in the Netherlands: The Need for Coordination Between the Public Health and the Curative Sector. J Public Health Manag Pract. 2018;24(1):18-25. 